\title{
A Fast Implementation of the Incremental Backprojection Algorithms for Parallel Beam Geometries ${ }^{\S}$
}

\author{
Chung-Ming Chen ${ }^{\dagger}$, Zang-Hee Cho ${ }^{*}$, Cheng-Yi Wang ${ }^{\dagger}$ \\ $\dagger$ Center for Biomedical Engineering, College of Medicine, NTU, Taiwan \\ * Dept. of Radiological Sciences, Univ. of California at Irvine, U.S.A.
}

\begin{abstract}
The Incremental backprojection algorithm is a fast backprojection approach requiring only $O(N)$ and $O\left(N^{2}\right)$ multiplications in contrast to $O\left(N^{2}\right)$ and $O\left(N^{3}\right)$ multiplications for the Shepp and Logan's backprojection algorithm in 2D and 3D backprojections, respectively, for each view. However, the improvement achieved by the Incremental algorithm in practice was not so significant as expected in theory due to inevitably visiting pixels outside the beam in the searching flow scheme originally developed for the Incremental algorithm.

To optimize implementation of the Incremental algorithm, an efficient scheme, namely, coded searching flow scheme, is proposed in this paper to minimize the overhead caused by searching for all pixels in a beam. The key idea of this scheme is to encode the searching flow for all pixels inside each beam. While backprojection, all pixels may be visited without any overhead due to using the coded searching flow as the $a$ priori information. The proposed coded searching flow scheme has been implemented on a Sun Sparc 10 and a Sun Sparc 20 workstations. The implementation results show that the proposed scheme is $1.45-2.0$ times faster than the original searching flow scheme for most cases tested. Compared to the conventional Shepp and Logan's backprojection algorithm, the proposed scheme may achieve 5-8 and 3 times speed-ups for 2D and 3D backprojection, respectively.
\end{abstract}

\section{INTRODUCTION}

Filtered-backprojection algorithms are the most widelyused approaches for reconstruction of computed tomographic (CT) images, such as X-ray CT and PET images. The main reason is that a filtered-backprojection algorithm may reconstruct an image much faster than an iterative algorithm. However, even for a conventional filtered-backprojection algorithm, i.e., using FFT for filtering and Shepp and Logan's algorithm for backprojection, it still needs about 40 seconds on a Sun Sparc 10 workstation to reconstruct a $256 \times 256$ image from 256 views of projection data. This speed might be acceptable for reconstructing a single slice of the CT image,

$\S$ This work was partially supported by National Science Council, R.O.C., under the contract NSC83-0420-E-002-009. but definitely not fast enough for multiple slices. For example, a patient who takes 32 slices needs to wait for more than 20 minutes to have all images reconstructed. It can be easily seen that much more time would be required for a larger size of images and for true 3D tomographic images, i.e., images reconstructed from not only horizontal projections but also from all other possible angles of projections.

To reconstruct an image in a reasonable time, various fast approaches have been proposed for a filtered-backprojection algorithm. Apparently, most researches have been concentrated on reducing backprojection time since, by using the well-established FFT, filtering takes much less time than backprojection. For backprojection, these approaches may be classified into three categories, namely, algorithmic improvement [1-2], dedicated hardware [3-5] and parallel processing [6-10]. While the last two categories counted on a higher computing power, the first one attempted to speed up backprojection by improving sequential backprojection algorithms. In general, it is expected that the last two categories promise a faster back-projection than the first category. However, improvement achieved by the first category may significantly reduce the cost of the hardware required by the last two.

The Incremental algorithm [2], belonging to the first category, is a fast backprojection algorithm proposed by Cho et al. based on the idea of transforming interdependency of pixel computations (position and value) into a set of incremental relations for each beam to minimize the computational redundancy for parallel beam geometries. Unlike another algorithm in the first category, called STRETCH[1], which is an approximated approach, the Incremental algorithm provides a not only fast but also accurate backprojection.

Theoretically, the Incremental algorithm may reduce the number of multiplications for 2D and 3D backprojections from $O\left(N^{2}\right)$ and $O\left(N^{3}\right)$ to $O(N)$ and $O\left(N^{2}\right)$, for each view., respectively. However, the improvement achieved by the Incremental algorithm in practice was not so significant as expected in theory. The primary reason is while searching for all pixels in each beam, overheads may be incurred due to inevitably visiting pixels outside the beam in the searching flow scheme originally developed for the Incremental algorithm [2]. For a $512 \times 512$ image, the overhead may amount to as much as $30 \%$ of pixel visitings in a view.

To optimize implementation of the Incremental algorithm, an efficient scheme, called coded searching flow scheme, is developed in this study to minimize the overhead caused by 
searching for all pixels in a beam. The key idea of this scheme is to encode the searching flow for all pixels inside each beam. While backprojection, all pixels may be visited without any overhead due to using the coded searching flow as the a priori information. To cope with the new overhead which may be caused by coding, an effective coding technique has been developed.

For ease of presentation, the searching flow scheme proposed in [2] will be referred to as on-line searching flow scheme in contrast to the coded searching flow scheme proposed in this paper. This paper is organized as follows. In Section II, the on-line searching flow scheme proposed in [2] is first briefly reviewed. Then, our new idea and implementation results for $2 \mathrm{D}$ and 3D backprojections will be presented in Section III and IV respectively. The conclusions are given in Section $V$.

\section{INCREMENTAL ALGORITHMS}

In order to realize the idea of updating pixel (voxel) values incrementally with additions only, the on-line searching flow scheme was proposed in [2] to determine all pixels in the same beam. For the 2D Incremental algorithm, the on-line searching flow scheme may be illustrated by Fig. 1. To find out all pixels in beam 1 , from the first pixel, i.e., $(0,0)$, the online searching flow scheme checks the pixels to the right and below and visit the one inside beam 1, i.e., $(0,1)$, in this case. Note that the pixels to be checked depend on the view angle, which was described in Table 1 of [2]. The same procedure repeats for all pixels. For the 3D Incremental algorithm, the possible directions to visit the next voxel are three instead of two as for the $2 \mathrm{D}$ case. However, in some cases, the visited pixels (voxels) are not inside the current beam. For example, at the pixel $(2,1)$, one can see that pixels $(2,2)$ and $(3,1)$ are both outside beam 1. Thus, the on-line searching flow scheme cannot but visit a pixel not inside the beam, which causes extra overhead for the Incremental algorithm. As a consequence, the actual speed up achieved by the Incremental algorithm in [2] is not so substantial as expected.

To see the efficiency of the on-line searching flow scheme for each view, we actually count the number of pixels visited and the number of pixels inside the beams, i.e., effective visits, for a $511 \times 5112 \mathrm{D}$ image. The results is illustrated in Fig. 2, in which visited denotes the number of pixels visited in each view and inside_beam the number of pixels inside the beam (refer to left vertical axis). In addition, the efficiency of the on-line searching flow scheme, i.e., inside_beam/visited, is also shown in Fig. 2 (refer to right vertical axis). The horizontal axis of Fig. 2 represents view numbers ranging from 0 to 511 , where the $i$ th view is at the angle of $i \times \pi / 512$. It can be easily seen that the efficiency of the on-line searching flow scheme varies dramatically from view to view. Clearly, it is least effective at the angles of $\pi / 4$ and $3 \pi / 4$ and as much as $30 \%$ of pixels visited are outside the beams in the searching flow at these two views.

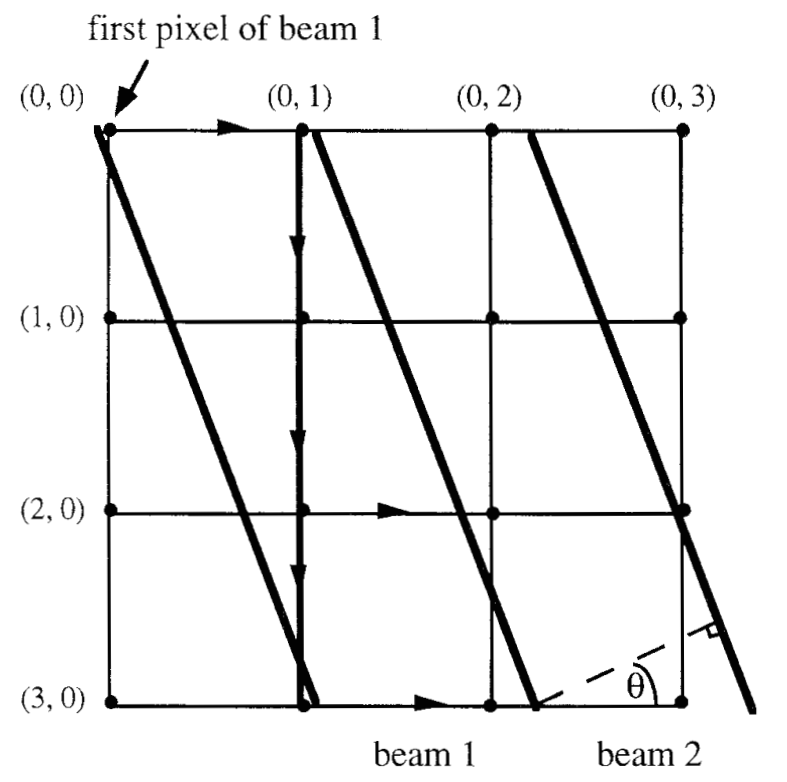

Figure 1. Searching flow in beam 1.

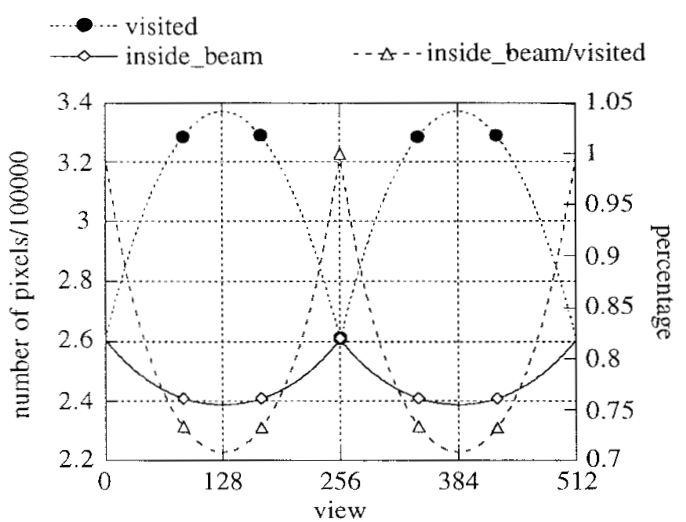

Figure 2. Efficiency of the on-line searching flow scheme.

To enhance the efficiency of the Incremental algorithm, a new scheme, namely, coded searching flow scheme, has been developed to alleviate the new overhead resulted from visiting pixels outside the beams in the searching flow.

\section{CODED SEARCHING FLOW SCHEME}

The basic idea of the coded searching flow scheme is to encode the searching flow path into path codes in advance and use the path codes as the a priori information during backprojection. Note that for each beam, only those pixels inside the beam will be encoded in the coded path. However, it is not difficult to imagine that encoding the searching flow path would incur another new overhead, i.e., decoding the 
coded path, though it can remove the overhead caused by visiting pixels outside the beams being searched. Also, it would need an enormous amount of memory to store the path codes.

Table 1. Possible directions of movements from one pixel to next pixel within the same beam in the $2 \mathrm{D}$ Incremental algorithm.

\begin{tabular}{|l|c|c|c|}
\hline & $\begin{array}{c}\text { horizontal } \\
\text { code: } 01\end{array}$ & $\begin{array}{c}\text { vertical } \\
\text { code: } 10\end{array}$ & $\begin{array}{c}\text { diagonal } \\
\text { code: } 11\end{array}$ \\
\hline $0 \leq \theta<\pi / 4$ & $\rightarrow$ & $\downarrow$ & $\searrow$ \\
\hline$\pi / 4 \leq \theta<\pi / 2$ & $\downarrow$ & $\rightarrow$ & $\searrow$ \\
\hline$\pi / 2 \leq \theta<3 \pi / 4$ & $\rightarrow$ & $\uparrow$ & $\nearrow$ \\
\hline $3 \pi / 4 \leq \theta<\pi$ & $\uparrow$ & $\rightarrow$ & $\nearrow$ \\
\hline
\end{tabular}

To minimize the overhead due to decoding the path codes and the amount of memory for storing path codes, three techniques have been employed in the proposed coded searching flow scheme. The first technique is to use $n$-bit codes for the $n$-D incremental algorithm, where $n$ is 2 or 3 . For $2 \mathrm{D}$ backprojection, since the possible directions of movements from one pixel to next pixel within the same beam are only horizontal, vertical and diagonal, at most 2 bits would be enough to encode the movements. The possible directions for different angles in 2D backprojection are listed in Table 1. Note that code 00 may be used as the end-of-beam flag. As an example, the path codes for the beam 1 in Fig. 1 is 11101001 , in which the first movement is coded in the rightmost two bits and the last movement in the leftmost two bits. Similarly, for 3D backprojection, seven directions are possible for movement from one voxel to the next one within the same beam, which may be encoded by 3 bits.

The second technique is to ensure that only $n$ bits are required while the area for backprojection is as small as possible. Ideally, backprojection needs to be carried out only inside the largest inscribed circle of the square image. However, if only the largest inscribed circle is considered, in some areas, the movement from one pixel to next pixel inside the beam might not be in any one of the expected directions in $2 \mathrm{D}$ or $3 \mathrm{D}$ backprojection. For example, in Fig. 3, since pixels 2 and 3 are outside the circle, even though they are inside the beam, they cannot be included in the coded path. As a result, moving from pixel 1 to pixel 4 is beyond the consideration in Table 1.

To make sure that all movements are in one of the three and seven directions for $2 \mathrm{D}$ and $3 \mathrm{D}$ backprojection, respectively, as the second technique, only the minimal area enclosing the largest inscribed circled covered by the beams in each view is backprojected. For instance, the beam-paved area for $\theta=0$ in 2D backprojection is illustrated in Fig. 4. Although this beam-paved area would include more pixels than the maximal inscribed circle, it can guarantee that $n$ bits would be enough for encoding all possible movements in the $n$-D backprojection.

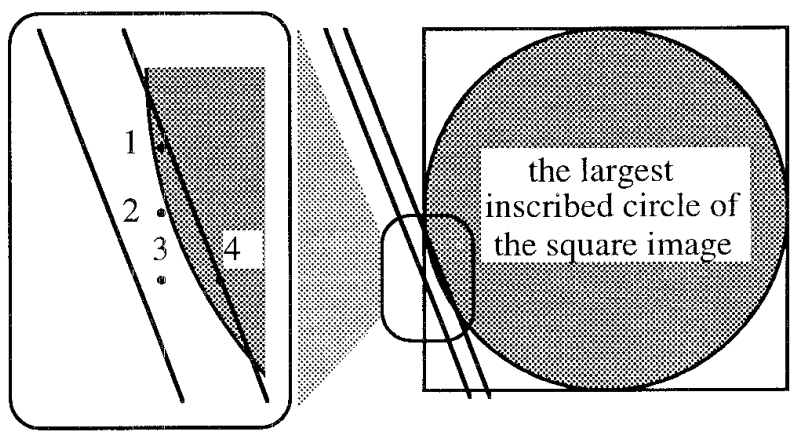

Figure 3. Movement from pixel 1 to pixel 4 is beyond the consideration in Table 1.

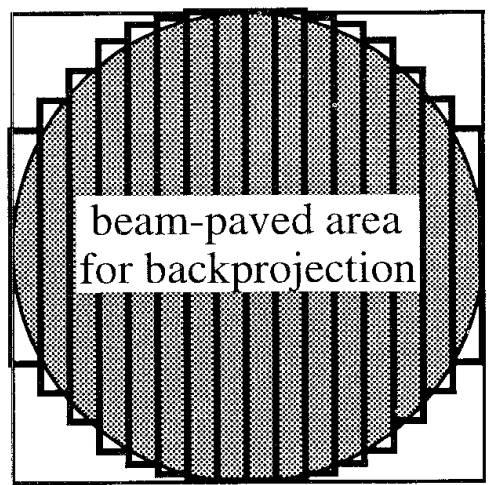

Figure 4. Beam-paved backprojection area for $\theta=0$ in 2D backprojection

To further reduce the amount of memory required to store all path codes, the third technique utilizes the symmetry of the searching flow paths. Consider the 2D backprojection. Suppose that the image size is $N \times N$ and the number of views is $N$, where $N$ is a power of 2. All views are numbered from 0 to $N-1$. There are $N$ projection data, i.e., $N$ rays, in each view. All rays in each view are numbered from 0 to $N-1$ and from left to right. Beam $i$ in a view is enclosed by ray $i$ and $(i+1)$ as defined before. Then, the searching flow path for the beam $i$ in view $j$, where $0 \leq j<\mathrm{N} / 4$, would be identical with that of

- beam $(N-2)-i$ in view $N / 2-j$,

- beam $i$ in view $j+N / 2$,

- beam $(N-2)-i$ in view $N-j$. 
That is, only one quarter of the path codes need to be stored. However, it should be noted that although these four beams share the same path codes, one should refer to Table 1 for the directions of movements for different views. The same idea may be applied to 3D backprojection in which only one eighth of the path codes need to be stored.

Based on these three techniques, one may easily generate the searching flow path codes by using Shepp and Logan's algorithm without actually doing interpolations. In addition to the paths, one may record the locations of the first pixels of all beams and their distances to the left rays since they are fixed for a given PET system and for the same size of images. While backprojection, it is suggested that among the three possible codes, code 10 should be checked first to minimize the checking time since it occurs more frequently than the other two in the 2D searching flow.

\section{IMPLEMENTATION RESULTS AND DISCUSSIONS}

To compare the on-line searching flow scheme and the coded searching flow scheme, the Incremental algorithm has been implemented with these two schemes on two different systems, namely, Sun Sparc 10 and 20 workstations. As a reference, the Shepp and Logan's algorithm has also been implemented. Four commonly-used images sizes were employed, i.e., $64 \times 64,128 \times 128,256 \times 256$ and $512 \times 512$, for $2 \mathrm{D}$ backprojection. For $3 \mathrm{D}$ backprojection, limited by the memory capacity of the available systems, the sizes of the images considered are $64 \times 64 \times 64,96 \times 96 \times 96,128 \times 128$ $\times 128$, and $160 \times 160 \times 160$. Figs. 5 and 6 illustrate the relative performance of every two algorithms on a Sparc 10 and a Sparc 20, respectively, for 2D backprojection. Figs. 7 and 8 illustrate the relative performance for $3 \mathrm{D}$ backprojection. In Figs. 5-8, Shepp denotes Shepp and Logan's algorithm, On-line the Incremental algorithm with the on-line searching flow scheme, Coded the Incremental algorithm with the coded searching flow scheme.

From Figs. 5-8, it is clear that the newly developed coded searching flow is superior to the on-line searching flow scheme. On both systems for 2D backprojection, the Incremental algorithm with the coded searching flow scheme can be more than 5-8 times faster than the Shepp and Logan's algorithm and more than 1.5 times faster than when the online searching flow scheme is employed. For 3D backprojection, the speed-ups, though smaller than for $2 \mathrm{D}$ cases, are still about 3 and 1.5 times, respectively. The reason why Shepp/Coded decreases as the image size increases for 2D backprojection may be ascribed to the insufficient cache memory in the Sparc machines employed and the increased array size for storing path codes. It is conjectured that if the workstation is equipped with a memory access technology comparable with its CPU technology, a better performance may be attained and the speed-ups would not decrease as the image size increases.

It is worth while pointing out that the overhead due to visiting pixels outside the beams being searched is at most $30 \%$ of all pixel visits (refer to Fig. 2 as an example). However, the improvement achieved by the coded searching flow scheme over the on-line searching flow scheme is far more than $30 \%$. It means that the coded searching scheme has offered some unexpected advantages. As a matter of fact, the biggest advantage of the coded searching flow scheme in addition to efficient visiting is the much easier programming than that for the on-line searching flow scheme. The reasons why the programming of the on-line searching flow scheme is more complicated are

- pairwise backprojection was employed to reduce the required searching effort to a half,

- it tried to end searching as soon as the searching flow reaches the boundary of the largest inscribed circle of the square image.

Another important advantage of the coded searching flow scheme is that it is more suitable for parallel processing than its counterpart. The reason is since all pixel visits are known beforehand and the computational load associated with all pixel visits are almost the same, it is quite easy to achieve balanced load distribution.

On the other hand, the only price to be paid by the coded searching flow scheme is a large amount of memory to store all path codes. As an example, it would need $970 \mathrm{~K}$ memory for an $512 \times 512$ image with 512 views. For a modern computer, it is believed to be acceptable. Therefore, the coded searching flow scheme is an ideal approach for the 2D Incremental algorithm. For the 3D Incremental algorithm, the required memory may be too large to be practical. However, this problem may be alleviated by further compressing the path codes.

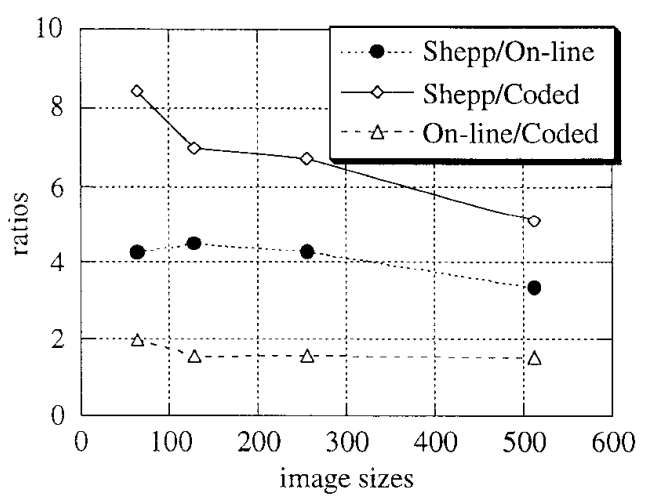

Figure 5. Relative performance on a Sparc 10 for $2 \mathrm{D}$ backprojection. 


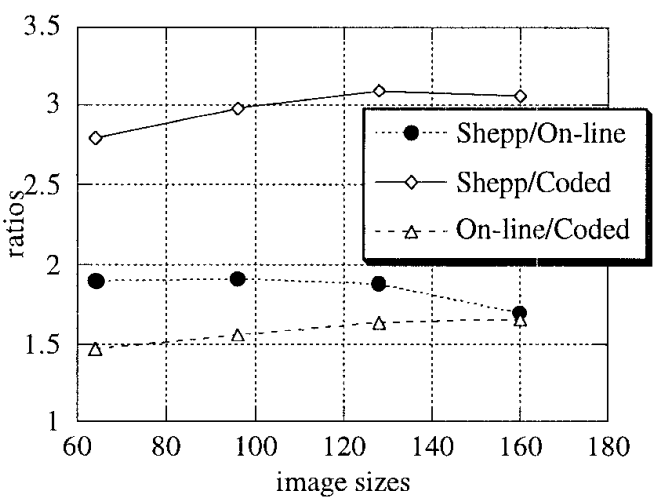

Figure 6 Relative performance on a Sparc 10 for 3D backprojection.

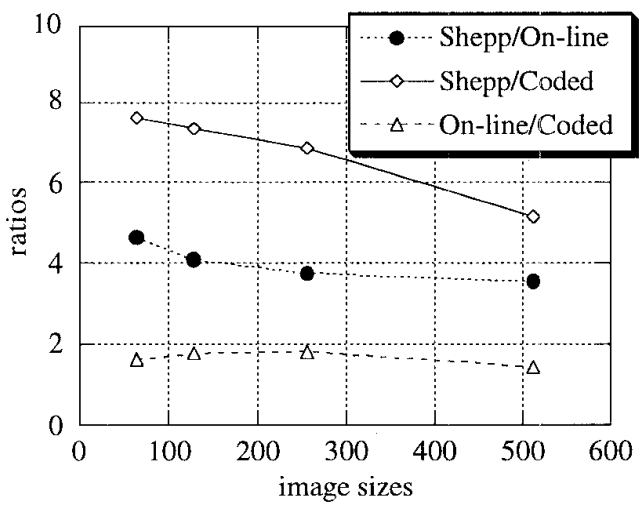

Figure 7. Relative performance on a Sparc 20 for $2 \mathrm{D}$ backprojection.

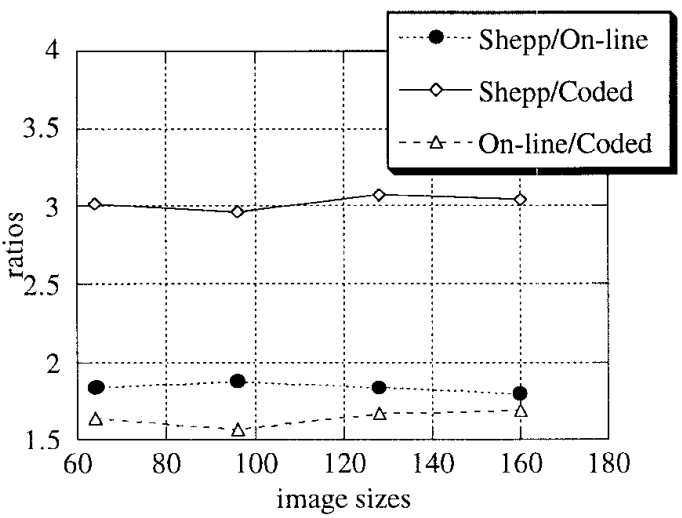

Figure 8. Relative performance on a Sparc 20 for 3D backprojection.

\section{CONCLUSIONS}

In the attempt to improve the Incremental backprojection algorithm, we have proposed a new searching flow scheme, called coded searching flow scheme, to minimize the overhead caused by searching for pixels in each beam. By encoding the searching flow into path codes and using the path codes as the a priori information while backprojection, the coded searching flow scheme has effectively eliminated redundant visits involved in the on-line searching flow scheme originally proposed in [2]. From the implementation results, we have shown that the coded searching flow is much more efficient than the on-line searching flow scheme. Since the proposed scheme may be programmed more easily, it also makes the Incremental algorithm more practical than before provided that the computer has enough memory. In addition, the coded searching flow scheme may be parallelized more efficiently than the on-line searching flow scheme since all information required for task partitioning is available before parallelization.

\section{REFERENCES}

[1] T. M. Peters, "Algorithms for fast back-and-re projection in computed tomography," IEEE Trans. Nucl. Sci., vol. NS-28, pp. 3641-3647, Aug. 1981.

[2] Z. H. Cho, C. M. Chen, and S. Y. Lee, "Incremental algorithm - a new fast backprojection scheme for parallel beam geometries," IEEE Trans. Med. Imaging, vol. 9, pp. 207-217, June 1990.

[3] C. J. Thompson and T. M. Peters, "A fractional address accumulator for fast backprojection," IEEE Trans. Nucl. Sci., vol. NS-28, pp. 3648-3650, Aug. 1981.

[4] Analogic Corporation, Wakefield, MA, Modular Image Processor(MIP), IP-300 Technical Manual, 1981.

[5] R. Hartz, D. Bristow, and N. Mullani, "A real-time TOFPET slice-backproject engine employing dual Am 29116 microprocessors," IEEE Trans. Nucl. Sci., vol. NS-32, pp. 839-842, Feb. 1985.

[6] C. W. Stearns, D. A. Chesler, and G. L. Brownell, "Three dimensional image reconstruction in the fourier domain," IEEE Trans. Nucl. Sci., vol. NS-34, pp. 374-378, Feb. 1987.

[7] S. Barresi, D. Bollini, and A. Del Guerra, "Use of a transputer system for fast 3-D image reconstruction in 3-D PET," IEEE Trans. Nucl. Sci., vol. NS-37, pp. 812-817, April 1990.

[8] M. S. Atkins, D. Murray, and R. L. Harrop, "Use of transputers in a 3-D positron emission tomography," IEEE Trans. Med. Imaging, vol. 10, pp. 276-283, Sept. 1991.

[9] W. F. Jones, L. G. Byars, and M. E. Casey, "Design of a super fast three-dimensional projection system for positron emission tomography," IEEE Trans. Nucl. Sci., vol. NS-37, pp. 800-804, April 1990.

[10] C. M. Chen, S. Y. Lee, and Z. H. Cho, "A parallel implementation of 3-D CT image reconstruction on hypercube multiprocessor," IEEE Trans. Nucl. Sci., vol. NS-37, pp. 1333 1346, June 1990. 